Cipango $\begin{aligned} & \text { Cipango } \\ & \text { Cahiers d'études japonaises }\end{aligned}$

15 | 2008

Guerre, colonialisme et commémoration

\title{
Les rapports entre les danses et les arts martiaux d'Okinawa. De la forme à l'identité
}

Jean-Charles Juster

\section{(2) OpenEdition}

1 Journals

\section{Édition électronique}

URL : https://journals.openedition.org/cipango/442

DOI : 10.4000/cipango.442

ISSN : 2260-7706

Éditeur

INALCO

\section{Édition imprimée}

Date de publication : 1 janvier 2008

Pagination : 273-278

ISBN : 978-2-85831-177-4

ISSN : $1164-5857$

\section{Référence électronique}

Jean-Charles Juster, « Les rapports entre les danses et les arts martiaux d'Okinawa. De la forme à l'identité », Cipango [En ligne], 15 | 2008, mis en ligne le 14 novembre 2011, consulté le 30 juin 2021 URL : http://journals.openedition.org/cipango/442 ; DOI : https://doi.org/10.4000/cipango.442

Ce document a été généré automatiquement le 30 juin 2021.

\section{(c) (7) (8)}

Cipango est mis à disposition selon les termes de la Licence Creative Commons Attribution - Pas d'Utilisation Commerciale 4.0 International. 


\title{
Les rapports entre les danses et les arts martiaux d'Okinawa. De la forme à l'identité
}

\author{
Jean-Charles Juster
}

\section{RÉFÉRENCE}

Les rapports entre les danses et les arts martiaux d'Okinawa. De la forme à l'identité, Thèse de doctorat soutenue par Jean-Charles Juster (Inalco, 2008), sous la direction de F. Macé.

1 Entre les mois de janvier 2004 et mars 2007, nous avons effectué plusieurs observations dans différentes écoles de danse de la ville de Naha et dans des hameaux de la partie Nord de l'île principale d'okinawa. Cette recherche visait à nous permettre de statuer sur les liens entre les danses et les arts martiaux de cette île, sujet revenant de façon récurrente au sein du monde chorégraphique local. Au-delà de ce travail, nous en sommes parvenu à réfléchir sur l'identité des Okinawanais exprimée au travers de cette relation entre danse et combat.

2 Notre thèse est articulée en trois mouvements intitulés « Aspects historiques et sociaux des danses et des arts martiaux d'Okinawa », «Observation et analyse de la présence du combat dans les danses des Ryūkyū », et « Danser martialement: identité par le corps et par rapport au corps ». Le premier suit une approche historique, le deuxième repose sur nos observations de terrain et propose des analyses du mouvement. Le troisième et dernier mouvement est quant à lui ethnologique.

3 Les danses d'Okinawa, parfois nommées Ryūkyū buyō, ont suivi les changements de la société de cette île. Lorsque l'on se penche sur leur histoire, on comprend qu'elles ont évolué, prenant place dans un cadre religieux, puis à la Cour du Royaume avant de connaître de profondes transformations dans le cadre d'un système fondé sur des représentations payantes. Notre recherche reposant sur les exécutants des danses et leurs milieux, nous avons insisté sur le fait que les pièces inscrites dans les rites étaient 
dansées par des personnes de sexe féminin qui étaient les desservantes des cultes. Les danses de cour étaient quant à elles l'apanage des nobles. Les gens du commun ne dansaient donc pas, en tout cas pas de façon institutionnalisée.

4 Nous avons également montré que l'émergence, à Okinawa et dans la métropole japonaise, d'études sur ce thème a entraîné la formation de la typologie utilisée de nos jours. Mais elle nous semble trop figée et ne rend pas compte de la réalité actuelle du monde chorégraphique d'okinawa où la danse telle qu'elle est pratiquée et pensée ne correspond pas forcément aux délimitations théoriques et aux définitions posées à son sujet.

5 Concernant l'histoire des arts martiaux insulaires, nous avons utilisé le peu de matériaux historiques (Kyūyō 球陽, Ōshima hikki 大島筆記) disponibles sur ce sujet. Nous avons ainsi expliqué que l'origine du karaté et des arts martiaux avec armes remonte au contact de la cour des Ryūkyū avec la Chine par l'intermédiaire des ambassades chinoises et de la présence de la communauté d'habitants du Fujian. Comme les danses, le combat à mains nues et celui avec armes étaient des activités réservées aux nobles. Avec l'entrée d'Okinawa dans l'ère moderne, elles connurent une phase d'expansion certaine et pénétrèrent la société de façon plus homogène. D'arts réservés à une élite issue de la cour, elles sont devenues des activités de loisirs dont le public n'a cessé de s'élargir.

6 Au cours de la seconde partie de notre travail, nous avons observé et analysé des séquences de bâtons sonbō, des danses masculines de cour, des danses modernes et des danses nettement marquées par les arts martiaux nommées mēkata et bu no mai. En nous appuyant sur la forme du mouvement martial, nous avons cherché à savoir si les gestes perçus comme combatifs le sont vraiment ou bien s'ils ne sont pas qu'une approximation d'une technique martiale.

7 En observant les séquences de bâtons, nous ne pouvons pas nous empêcher de penser au combat au bâton. Néanmoins, celui que l'on voit le plus souvent à l'heure actuelle ne leur ressemble pas. Certes, les sonbō que nous avons observés ont un aspect parfois spectaculaire, mais cela ne les empêche pas d'être liés au combat. Nous pensons que ces formes des campagnes, en fait peu sophistiquées et techniquement assez inférieures au combat au bâton des dōjō qui est, lui, clairement définis par des experts chinois, sont le reflet de ce qu'avait pu être le combat avec cette arme dans les temps anciens de l'île.

8 Les danses masculines que nous avons analysées comportent bien des éléments agonistiques. Mais comme nous le présupposions, ceux-ci ne se trouvent pas tout à fait dans les danses des villes, en tout cas pas d'une façon aussi prononcée que les spécialistes le disent souvent. Il nous semble en effet que la démarche esthétisante qu'ont connue de façon générale les danses de cour données à Naha durant le xix siècle a entraîné des modifications de certaines formes de leurs techniques de karaté. Par contre dans les hameaux, les danses sont toujours réalisées correctement, ce qui nous fait dire qu'elles ont gardé leur intégrité, peut-être parce que dans les campagnes cette recherche du beau n'a pas eu lieu.

9 Il est néanmoins intéressant de se demander pourquoi les spécialistes qui concentrent leur travaux sur les danses des écoles des villes ne voient pas cette altération. Cela s'explique peut-être par le fait qu'ils n'essayent pas d'analyser si les techniques de karaté présentent dans ces danses en sont réellement ? Ce qui sous-entendrait qu'ils se laissent en fait guider par la rumeur qui veut que le karaté soit présent dans ces pièces chorégraphiées, auquel cas il s'agirait d'un défaut d'analyse certain. Une autre 
explication serait qu'ils sont conscients de ces différences, mais qu'ils ne veulent pas s'opposer à ce que la tradition véhicule et en ce sens critiquer leur propre culture. De plus, en restant dans cette ligne officielle, ils apportent un particularisme certain aux danses d'Okinawa qui sont ainsi nettement différenciées de celle de la métropole.

Des danses issues du genre moderne comportent également des mouvements martiaux. La pièce Hatuma bushi (pour sa version moderne) nous montre que le chorégraphe Iraha Inkichi a tenté de se servir du karaté pour construire son œuvre. Cependant, il n'est pas parvenu à restituer complètement les formes des techniques de karaté, car il n'était pas lui-même un pratiquant. A travers cet exemple, nous comprenons la place relativement importante du karaté dans la culture d'Okinawa puisqu'un chorégraphe a jugé bon d'un point de vue artistique de s'en inspirer pour dépasser les limites entre le monde de la danse et celui des arts martiaux.

11 La pièce Hamichidori est une pièce créée assez récemment (1897), mais qui comporte des techniques traditionnelles des danses des Ryūkyū. Trois de ses mouvements se retrouvent dans la Motobu-ryū uduntī, une école traditionnelle d'arts martiaux. Ainsi, à travers ce rapport entre cette danse et cette école martiale, nous pouvons envisager que c'est la danse qui a influencé l'art martial, contrairement aux danses classiques masculines ou à la pièce Hatuma bushi moderne. Il est aussi possible de voir dans ce rapport un fond commun où la danse et les arts martiaux n'étaient pas différenciés à une époque que nous estimons aller jusqu'aux XIII ${ }^{\mathrm{e}}$-XIV ${ }^{\mathrm{e}}$ siècles.

12 Yotsudake est une danse de cour féminine. La version bien particulière de cette pièce que nous avons observée au hameau de Namizato repose sur une gestuelle où est figuré le maniement martial d'un bâton. Cette version est différente de l'interprétation courante que l'on donne dans les villes comme dans les hameaux. Grâce à cette danse, nous voyons que certaines interprétations de danses qui ne devraient normalement pas comporter d'éléments agonistiques au regard de leurs origines, sont justement l'occasion d'observer l'influence d'un art martial, dans ce cas précis le combat au bâton.

13 Les mēkata et les bu no mai mettent en avant d'autres éléments utiles à notre étude. Premièrement, même s'il s'agit officiellement de danses, les individus sur scène sont des pratiquants, à des degrés plus ou moins forts, d'arts martiaux. Il ne s'agit plus de danseurs, comme c'est le cas pour les danses masculines de cour ou les danses modernes, ni d'individus recevant une instruction rapide quelques semaines avant le jour du matsuri, comme c'est le cas pour les sonbō. Leurs gestes ne sont donc pas des représentations, même inconscientes, de techniques d'arts martiaux, ni des répétitions, sans doute non réfléchies, de ce que leurs aînés ou leurs maîtres leur ont montré. Deuxièmement, ce ne sont pas tant des danses que des kata situés dans un contexte festif (costume, musique d'accompagnement, scène) et créés pour l'occasion, comme nous l'avons expliqué. Il est donc étrange que les terminologies employées pour les désigner mettent davantage l'accent sur la danse que sur l'aspect martial pourtant prépondérant. De fait, le spectateur est ainsi mal informé et s'attend davantage à assister à une danse qu'à un kata de karaté ou de combat au bâton. Pourtant, quand dans les hameaux des suites de mouvements de tridents sai et de karaté sont exécutés, il ne fait aucun doute qu'il s'agit là de kata, et personne ne les dénomme bu no mai (trident) ou mēkata. Est-ce parce qu'ils ne le sont pas sur une scène et qu'une musique spéciale n'est pas alors jouée ? Sans doute, tant il semble exister une différence, nous semblant assez superficielle, entre spectacles donnés sur une scène, et perçus comme étant de vrais divertissements hérités de ceux de la cour, et spectacles réalisés à même 
le sol, donc ressentis comme étant plus populaires et donc d'un niveau qualitatif moins élevé. Pourtant, les spectacles à portée martiale : kata, bu no mai ou mēkata, sont de même qualité, indépendamment du lieu où ils prennent place.

Nous avons voulu dans cette dernière partie mettre en rapport nos conclusions relatives à la présence d'éléments martiaux dans les danses d'Okinawa avec les théories sur les techniques du corps.

Nous avons expliqué tout d'abord que le corps est porteur de valeurs. Certains spectacles que nous avons observés sont associés à une culture classique, d'autres à une culture populaire. Une segmentation se fait ainsi jour : d'un côté, il y a des corps jugés capables d'accomplir des pièces de théâtre et des danses masculines, de l'autre, ceux qui ne le peuvent pas sont contraints de réaliser des sonbō. Il y a une vision dualiste : certains corps peuvent et d'autres ne peuvent pas.

16 Nous avons vu que les habitants d'Okinawa ont des raisons de danser martialement qui s'expliquent par la symbolique (conjuratoire, combattante) ou par l'appartenance territoriale de ces danses. Tous ces facteurs renvoient à l'identité, et, en ce sens, nous montrent que danser avec des éléments martiaux va au-delà de la simple expression corporelle. Il y a un message derrière ces actions. Il n'est peut-être pas des plus explicites, mais appréhendé au travers du prisme des différentes situations que nous avons détaillées ou des hypothèses que nous avons émises, il devient plus compréhensible : danser martialement montre que le corps est un vecteur d'identité. La théorie que nous émettons concernant l'importance du corps dans le champ social se vérifie donc: en étudiant au plus près le corps à travers la question des rapports entre les danses et les arts martiaux, nous percevons les imbrications que les danses et les arts martiaux ont avec la société d'Okinawa, ce qui nous permet de mieux comprendre les comportements des habitants.

17 Cette dernière partie nous a également permis de préciser notre position face à la relativité des études liées au corps. Nous sommes en effet conscient que le corps touche à la fois à l'objectivité et à l'extrême subjectivité. Il constitue un élément concret, et est commun à tous les hommes, mais, de façon parallèle, il produit des signaux, est le support de mouvements et est l'objet de visions bien précises dont les interprétations sont multiples. Le corps, situé à la frontière du tangible et du ressenti, du charnel et de l'imaginé, nous montre qu'il est le produit des représentations que les sociétés ont de lui. Son identité se situe donc elle aussi au croisement de plusieurs champs (présupposés idéologiques, croyances religieuses, constituants physiologiques) touchant à la fois à l'objectivité et à la subjectivité.

18 Nous avons ensuite insisté sur le fait que les rapports entre le combat et la danse ne sont pas propres à Okinawa, mais qu'ils sont au contraire un phénomène commun à l'ensemble des sociétés humaines. C'est le cas de la métropole japonaise avec le nō et le kabuki qui comprennent des formes de corps provenant des écoles de combat classiques (sabre, fauchard, lance). C'est également le cas en Indonésie, en Afrique où des sociétés traditionnelles subsistent de façon beaucoup plus marquée. A la différence $\mathrm{du}$ Japon, ces qualités agonistiques qu'entretient la danse y ont une fonction et une utilité immédiate : la chasse. Au Japon, un danseur de nō sachant se servir d'un sabre, ou une danseuse de kabuki qui a appris le maniement du fauchard n'en aura pas l'utilité dans sa vie quotidienne.

Les mēkata sont à ce propos particulièrement intéressants. Il nous semble être des kata de karaté ou de combat au bâton réalisés dans le cadre de divertissements populaires. 
Mais au-delà de ce cadre folklorique, ils sont aussi populaires parce qu'à l'heure actuelle les arts martiaux font partie du patrimoine culturel local et qu'ils sont pratiqués par tous, sans distinction de classes sociales. Les mēkata sont donc l'incarnation d'un glissement du monde savant vers le domaine populaire. D'ailleurs, nous pensons que les habitants des hameaux sont peut-être influencés par la ruralité de leur propre milieu pour agir ainsi. Comme pour justifier la présence d'un divertissement dont les origines ne sont pas populaires, mais qui est pourtant actuellement uniquement donné dans un environnement qui l'est fortement, les habitants établissent une séparation nette entre aspect savant et aspect populaire et mettent en avant le second.

Notre thèse sur les danses d'Okinawa, où nous avons voulu nous attacher au corps et à ce qu'il véhicule contrairement à la majeure partie des travaux actuels, est un premier essai d'étude des techniques du corps au Japon. Il nous a semblé essentiel d'aller au contact des Okinawanais, de les observer danser, de les entendre parler de leurs traditions pour dépeindre cette société qui est le plus souvent l'objet de travaux historiques, mais dont les études ethnologiques sont malheureusement rares en Europe. La danse et les arts martiaux sont capables de nous aider à comprendre comment fonctionne la société d'Okinawa, et au-delà à mieux appréhender sa place au sein du Japon contemporain. 